\title{
Successful Pregnancy Outcome Within Short Period of Remission From Choriocarcinoma Treated with Single Agent Chemotherapy - A Case Report
}

\begin{abstract}
Gestational trophoblastic tumour/disease (GTT/GTD) is unique in cancer biology in that they result from aberrations of either a normal or an abnormal pregnancy. The most common antecedent pregnancy event to GTT is a complete or partial hydatidiform mole (HM). However, persistent trophoblastic disease or choriocarcinoma can follow a complete hydatidiform mole with an incidence of approximately $8 \%$, and after a partial hydatidiform mole with an incidence of approximately $0.5 \%$. The exact proportion of cases of hydatidiform mole transforming to choriocarcinoma cannot be clearly estimated, approximately $3 \%$ to $5 \%$ of cases of complete hydatidiform mole, 1-3 Therefore, all patients with GTT need to be monitored so that the small proportion of persistent mole or choriocarcinoma can receive prompt treatment and elimination of their disease. The recommendation stands as strict avoidance of pregnancy for at least one year after treatment of molar pregnancy or low risk non-metastatic gestational trophoblastic tumour.
\end{abstract}

Key words: pregnancy, sholt remission from chemotherapy, choriocarcinoma

\section{Introduction}

Gestational trophoblastic tumour/disease (GTT/GTD) is frequently cured with chemotherapy. Fertility is usually preserved following chemotherapy in these women but little is known about the risk of disease relapse or damage to the foetus in women who conceive soon after completing treatment. The advice to avoid pregnancy for 1 year post-chemotherapy in women with GTT has been determined by theoretical risks rather than solid clinical evidence of risk to the mother and foetus. ${ }^{1}$ The greatest risk of tumour relapse is within the first year of remission and early pregnancy can compromise both the surveillance systems used to detect relapse as well as the safe institution of subsequent therapies. In addition, it is possible that the hormonal changes associated with pregnancy could in some instances actually promote early recurrence. It is thought that this period allows ova which have been damaged by chemotherapy to either undergo

1. Dr. Asma Habib FCPS, Resident Assistant Professor, Department Of Obstetrics \& Gynaecology, Bangladesh Medical College and Hospital Prof

2. Dr. Md. Mofazzel Hossain, FCPS (Medicine), FRCP, FACP ( USA), Fellow in Med Oncology, Trained in NUH ( Sing), Professor of Oncology, department of Oncology, Bangladesh Medical College and Hospital.

3. Dr. Fauzia Jahan, M Phil (Histopathology), Assistant Professor, Department of Pathology, Bangladesh Medical College and Hospital Name and address of the author to whom correspondence regarding manuscript to be made and to whom request for reprint should be addressed:

Corresponding Author

4. Dr. Asma Habib, House: 74 (502), Road no: 8/A, Dhanmondi Residential Area, Dhaka-1209, e-mail: asma.habib54@yahoo.com
Pregnancy during this period of surveillance interferes with the sequential monitoring of abnormal trophoblastic activity by serum beta-human chorionic gonadotrophin levels and relapses become difficult to detect. The effect of single agent or combination chemotherapy on the totipotent oocytes usually wavers away during the recommended period of contraception. But in cases of pregnancy during the period of surveillance certain factors have been found to be associated with increased risk of relapse and teratogenic effects of the offspring. High risk/score (according to the FIGO scoring system) and advanced stage Gestational trophoblastic tumour (GTT), short interval between pregnancy and remission from combination chemotherapy, poor compliance during the antenatal follow-up are linked with detrimental maternal and foetal outcome. Here we report a case of successful pregnancy outcome in a patient who conceived within 3 months of remission from choriocarcinoma treated by methotrexate as evidenced by 2 consecutive negative $\beta$-human chorionic gonadotropin ( $\beta$ - $h C G$ ) values.

degeneration/atresia or repair, reducing the risk of foetal malformation and/or spontaneous abortion. Here we report a case of successful pregnancy outcome in a patient who conceived within 3 months of remission from choriocarcinoma treated by methotrexate as evidenced by 2 consecutive negative $(0.00 \mathrm{mIU} / \mathrm{ml}) \beta$-human chorionic gonadotropin $(\beta-\mathrm{hCG})$ values. She had a relatively uneventful antenatal period and delivered a male child by caesarean section at 36 completed weeks for obstetric indication (breech presentation with foetal distress and oligohydramnios). In the subsequent follow-up period of 9 months she remained symptom free and showed no evidence of tumour relapse.

\section{Case report}

A 19 year old primigravida presented with a history of 16 weeks of amenorrhoea and per vaginal bleeding on $19 / 9 / 2009$ at a local maternal and child health care delivery centre. On examination, her blood pressure was recorded $165 / 105 \mathrm{~mm}$ of $\mathrm{Hg}$ with ++ bed side urine albumin and uterine height corresponded to 24 weeks of gestation; markedly enlarged for the duration of amenorrhoea. Antihypertensive medication in the form of tablet Methyl-dopa was instituted with advice for an urgent ultrasonogram for evaluation of the pregnancy status. Ultrasonogram on 23/9/2009 revealed grossly enlarged uterus $(19.7 \times 13.2 \mathrm{~cm})$, cavity containing large amount of homogenous collection with numerous small anechoic spaces. No foetal part was seen. Both ovaries were found to be enlarged with multiple cysts. The comment was complete molar pregnancy with bilateral theca luteal cysts (Figure: 1). The patient was referred to Rangpur Medical College and Hospital for management. There was no evidence of metastasis beyond the uterus from the time of 
presentation until evacuation. She underwent suction and evacuation on $3 / 10 / 2009$. Serum $\beta$-hCG 1 month postevacuation was $13,302 \mathrm{mIU} / \mathrm{ml}$. She remained amenorrhoeic for 4 months following that evacuation. But the patient developed irregular per vaginal bleeding from March, 2010 and was referred to Bangladesh Medical College Hospital for further management. Ultrasonogram revealed thickened endometrium $(20 \mathrm{~mm})$ with small echogenic irregular foci near the fundus giving the suspicion of persistent molar tissue with $\beta$-hCG value of $>5,000 \mathrm{mIU} / \mathrm{ml}$. Evacuation and curettage was performed on $3 / 5 / 2010$. Histopathology of the endometrial tissue revealed choriocarcinoma composed of anaplastic syncytiotrophoblasts. No chorionic villi were seen in the background of massive haemorrhage and necrosis. (Figure: 2, 3) Serum $\beta$ - hCG was repeated 7 days after evacuation and found to be $1,744 \mathrm{mIU} / \mathrm{ml}$. She was then referred to the department of Oncology and single agent chemotherapy with Methotrexate and Folinic acid (MTX/FA) was started from seventh day post-evacuation, at two weekly interval for 6 cycles. Serum $\beta$-hCG values declined from 1,744 to $7.3 \mathrm{mIU} / \mathrm{ml}$ after the third cycle of chemotherapy. She had two consecutive negative $(0.00)$ reports on July and August, 2010. The patient was monitored in each cycle with prior complete blood count, serum electrolyes and $\beta$-hCG levels. The patient tolerated the chemotherapy without much difficulty. There was no clinical or biochemical evidence of relapse or persistence of trophoblastic disease with two consecutive zero values of $\beta$-hCG within the following 3 months. She had successfully completed 6 cycles of chemotherapy on $8 / 8 / 2010$. She resumed to have regular menstrual cycles from the third cycle for 3 subsequent months. But on 8th November 2010 she was found to have elevated $\beta$-hCG values of $26,525 \mathrm{mIU} / \mathrm{ml}$ and was already amenorrhoeic for $5+$ weeks. Trans-vaginal ultrasonogram revealed $5+$ weeks of early intra-uterine pregnancy with apparently normal looking trophoblastic ring and normal sac outline. The patient was referred to the outpatient department of Gynaecology and Obstetrics from Oncology department. As she had conceived after two consecutive negative $\beta$ -hCG and sonography revealed no obvious trophoblastic alteration even in that very early gestation with $\beta$-hCG values corresponding to normal pregnancy for that gestational age and the patient strongly desired to continue upon informed consent, the decision for continuation of this high risk pregnancy was taken. The patient was advised for strict coherence to obstetrician and she complied with that. Her pregnancy was monitored with routine antenatal investigations and clinical parameters such as weight gain, blood pressure and fundal height. The serum $\beta$-hCG values were repeated at $15,22,32$ weeks with values corresponding to normal trophoblastic activity $(49,017,24,308$ and $29,294.3 \mathrm{mIU} / \mathrm{ml}$ respectively). She was empirically put on folic acid supplementation, low dose aspirin and high-dose calcium from early gestation and had received steroid prophylaxis at $34+$ weeks. Anomaly scan at 21 weeks revealed no gross anomaly. She developed less foetal movement from $35+$ weeks with declining amniotic fluid index(AFI from 14 to 5) over the previous five weeks. She underwent emergency lower uterine Caesarean section at 36 weeks for non-reassuring foetal status (biophysical profile score 7) and breech presentation. The patient delivered a male baby of 2.2 kilograms. Upon immediate resuscitation and intensive care the baby had a subsequent uneventful neonatal course.

The placenta, cord and membrane were grossly unremarkable and the placenta was sent for histopathological examination which revealed atypical changes in the trophoblastic lining of few villi, but otherwise normal study. Post LUCS $\beta$-hCG value was $988 \mathrm{uIU} / \mathrm{ml}$ and declined to undetectable levels in the subsequent twice weekly follow-ups during puerperium. Follow -up visit at 9 months postdelivery demonstrated no clinical or biochemical evidence of disease relapse.

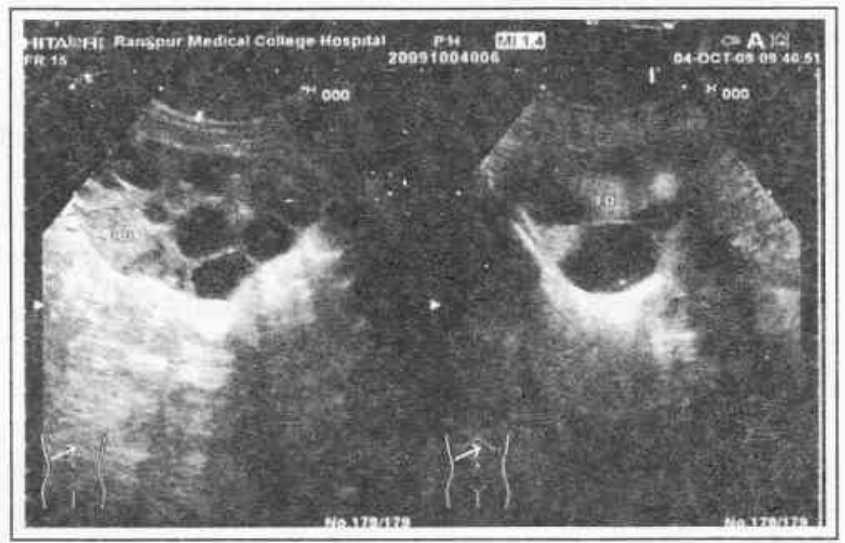

Figure: 1

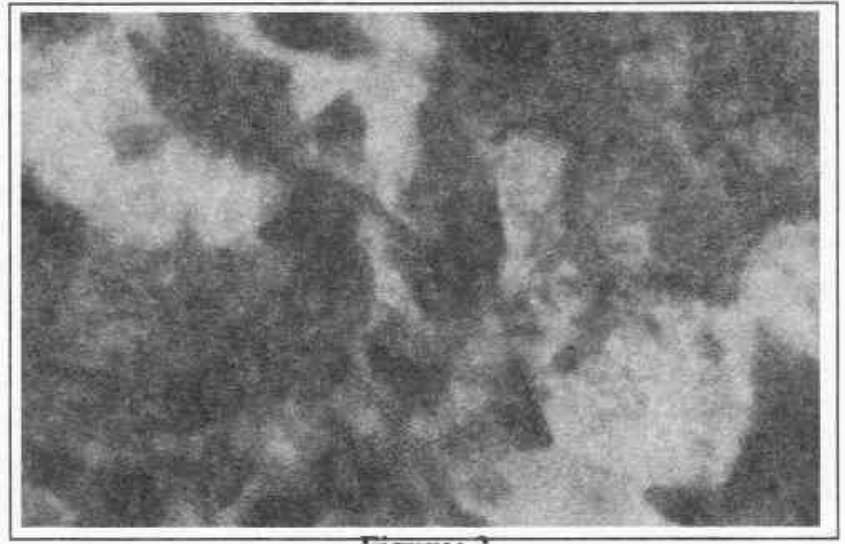

Figure: 2 


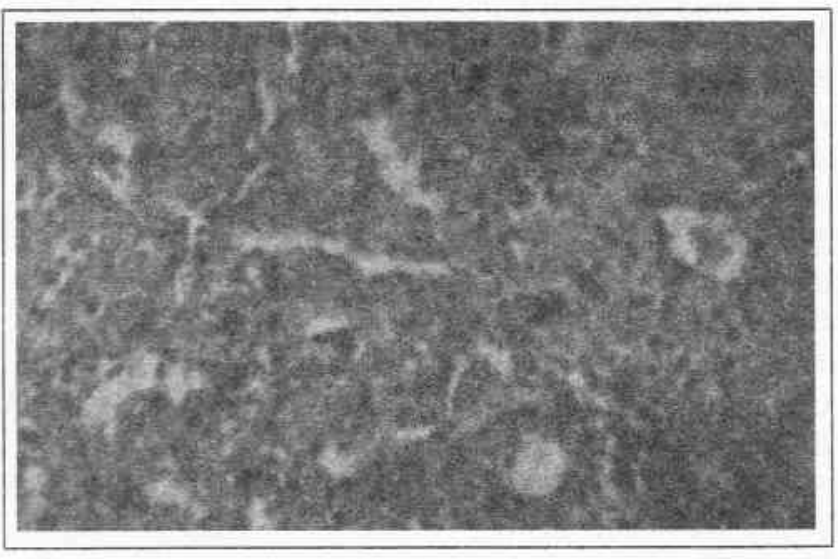

Figure: 3

\section{Discussion}

HM is the most common GTD. The incidence varies from 1 in 125 to 1 in 1500 deliveries; higher incidence is noted in women younger than 20 years and older than 40 years, in nulliparous women and in patients of low socioeconomic status. Blood group A women impregnated by group $\mathrm{O}$ men have a 10 fold greater risk of developing choriocarcinoma than group A women impregnated by group A partners.4 In this particular report the antecedent gestational event was complete hydatidiform mole and the patient was only 19 years of age .Both partners had blood group A. Hyper-emesis gravidarum, disproportionately enlarged uterine height, anaemia are common presenting features of complete mole. ${ }^{4}$ Rarely patients of complete mole present with early onset of severe pre-eclampsia and features of hyperthyroidism. Pre-eclampsia in first trimester or early second trimester has been said to be pathognomonic for mole, seen in $10-20 \%$ of patients ${ }^{5}$. This patient presented at 16 weeks of pregnancy with classical symptoms of severe pre-eclampsia as evidenced by elevated blood pressure and protienuria. Choriocarcinoma is a tumour composed both of cyto-trophoblastic and syncitio-trophoblastic cells. It is an unusual tumour in that it stimulates virtually no stromal reaction and is therefore essentially a mixture of haemorrhage and necrosis with tumour cells scattered within the mass. Tumour cells can be scanty and present problems of pathological interpretation. Therefore a histopathologic diagnosis of choriocarcinoma in any site is an indication for prompt treatment, as in this case. ${ }^{1-3}$ This patient received single agent chemotherapy with Methotrexate (MTX) within 7 days postevacuation as the curettage reported as choriocarcinoma. Methotrexate or dactinomycin is recommended for malignant non-metastatic or low risk GTD and also for poor compliant patients apprehended to be lost during follow -up as prophylaxis. Multiple agent chemotherapy (MAC/EMACO regimen) is used where resistance to single agent is anticipated and in high FIGO score patients.3 FIGO score of this particular case is 6; that is low risk non-metastatic. But the histologic evidence of choriocarcinoma warranted special care for the manage ment of this case. Single agent chemotherapy methotrexate (MTX) was successfully completed at 14 days interval at $50 \mathrm{mg} / \mathrm{m} 2(\mathrm{D} 1,3,5,7)$ with alternative folinic acid (FA) rescue( $\mathrm{D} 2,4,6,8)$ for 6 consecutive cycles. Early pregnancy following chemotherapy for GTT does not increase disease relapse. Single agent chemotherapy had no effect on rates of miscarriage, still birth or congenital malformation according to the review of records of 1532 patients treated for persistent gestational trophoblastic tumours at Charing Cross Hospital, U.K. between 1969 and1998.5 Relapse rates were $2 \%$ (3 out of 153 ) and $2.5 \%$ ( 2 out of 77 ) for single agent and Multi-agent group respectively compared to $5 \%$ and $5.6 \%$ in the comparative non-pregnant groups. Outcomes of 230 early pregnancies: $71 \%$ delivered at full term, 15\% ended in deliberate termination, $11 \%$ had spontaneous abortions, $1.3 \%$ had new/recurrent hydatidiform mole and 1\% stillbirths. Early pregnancies were more common in the single agent group $(\mathrm{P}<0.001)$, but spontaneous miscarriages and terminations were more common in the multi-agent group ( $\mathrm{P}=0.04$ and 0.03 , respectively). Of the full term pregnancies, three $(1.8 \%)$ babies were born with congenital abnormalities. ${ }^{5}$ Though it is still recommended to avoid pregnancy within 12 months of completing chemotherapy, those that do conceive can be reassured of a likely favourable outcome and not at increased risk of relapse. ${ }^{4}$ During antenatal check-up of this particular care certain points were emphasized at each visit. Correspondence of height of the uterus with the period of amenorrhoea, $\beta-\mathrm{hCG}$ values at different trimesters along with their comparison to normal levels, sonographic ehotexture of the placenta and foetal growth percentiles with exclusion of anomalies were the special precautions taken antenatally. Folic acid supplementation, low dose aspirin and high dose calcium were instituted from early gestation to prevent preeclampsia. 6 Serum $\beta$-hCG values were monitored quantitatively to detect the earliest evidence of abnormal trophoblastic activity beyond the normal values at 15, 22 and 32 weeks of gestation. The levels corresponded to normal values for each week of gestation.Prophylactic steroid therapy with Dexamethasone was given at 34 weeks of pregnancy in the anticipation of any emergency premature termination. The gradual but marked decline of AFI from $31+$ weeks over the following two weeks indicated placental failure which led to an emergency caesarean section at $36+$ weeks of pregnancy. Breech presentation was another indication for an abdominal delivery in this primiparous lady, thereby avoiding the risk of breech vaginal delivery in such a valuable pregnancy. This patient was exposed to MTX during periconceptional period, that is 3 months prior to conception, but the child exhibited no congenital malformation. From this perspective, methotrexate embryopathy, known as aminopterin syndrome has been described in several reports in cases of early ( first trimester ) exposure of MTX in pregnancy .The syndrome includes holoprosencephaly, hypertrichosis, facial dysmorphism (cleft lip/palate), mesomelias 
(short forearms) and long eyelashes. ${ }^{7,8}$ The incidence of congenital heart abnormalities was higher for patients undergoing combined chemotherapy during this vulnerable period than in the general population. ${ }^{9}$ There are certain controversial issues in the comprehensive management of this case. One, when to declare the patient of choriocarcinoma disease-free and two, upon what criteria to rule out the necessity of hysterectomy in such a case, last of all ,the risk of recurrence of choriocarcinoma in subsequent conceptions or whether she should be debarred against future childbirth. Regarding the first issue, there is remote possibility of mole/choriocarcinoma to present with overt metastatic features even several decades following a normal delivery. ${ }^{4}$ Therefore each case of choriocarcinoma needs life-long surveillance, even following hysterectomy. But certain factors favour a higher recurrence /relapse risk in patients with GTD such as antecedent term pregnancy, delayed diagnosis (more than 6 months), pretreatment $\beta$-hCG $\geq 1,000 \mathrm{mIU} / \mathrm{ml}$, metastasis beyond lungs at the time of diagnosis, persistent or rising $\beta$-hCG despite chemotherapy. Such high risk patients should be kept under meticulous surveillance protocol and strongly advised against conception. ${ }^{10}$ The incidence of a further/recurrent molar pregnancy is 1 in $76 .^{1,5}$ Previous history of choriocarcinoma was an indication of elective caesarean/ peripartum hysterectomy prior to the era of highly sensitive chemotherapeutic options, particularly Methotrexate. ${ }^{11}$ Hysterectomy is advocated in multiparous and elderly women, but rarely recommended in pauciparous patients considering the efficiency of to MTX or multi-agent regimens to eliminate trophoblsastic tissues. Patients with history of choriocarcinoma treated with chemptherapy had comparable survival rates to those undergoing hysterectomy at more recent time points. ${ }^{12,13}$ Combined oral contraceptive pill is the preferred choice of contraception for HM patient atleast for two years to continue serological surveillance with $\beta-\mathrm{hCG}^{2,4}$ Preconceptional planning and re-evaluation of disease status are essential if future pregnancy is contemplated. Another issue in management is the psychological impact of the disease on the patient. Undergoing life-long surveillance, being aware of its recurrence risk and bearing the womb itself may produce immense stress which needs to be addressed and mitigated as much as possible. ${ }^{14}$

\section{Conclusion}

In conclusion, it must be emphasized that strict contraceptive advice with combined oral contraceptives are strongly recommended for all patients recovering from molar pregnancy whether they receive chemotherapy or not. In case of early conception following chemotherapy counseling is essential for the patient and partner regarding the risk of relapse/metastasis and effects of chemotherapy on the unborn child. With the known risks pregnancy may be continued upon informed consent under strict feto-maternal supervision and oncologic consultation.

\section{References}

1. Ilancharan A, Singh P. Update of molar pregnancy and choriocarcinoma. Sing Med J.1989.30:473-475.

2. Bagshawe KD. Risk and prognostic factors in trophoblastic neoplasia. Cancer . 1976,38: 1373-1385.

3. Newlands ES. Clinical management of trophoblastic disease in the United Kingdom. Current Obstetrics \& Gynaecology .1995; 5: 19-24.

4. Aghajanian P. Gestational trophoblastic diseases. Current diagnosis in obstetrics and Gynaecology. 1995.9th Edition; 884-895.

5. Kawano $H$, Nose $\mathrm{R}$, Bagshawe KD . Risk and prognostic factors in trophoblastic neoplasia. Cancer 1976; 38: 13733-1385.

6. Magee LA, Helewa M, Moutquin JM, von Dadelszen P, Hypertension Guideline Committee, Society of Obstetricians and Gynaecologists of Canada. Prediction, prevention, and prognosis of preeclampsia. In: Diagnosis, evaluation, and management of the hypertensive disorders of pregnancy. J Obstet Gynaecol Can 2008 Mar;30(3 Suppl 1):S16-23.

7. Ahmed Z S, Alabbae M M. S, Omar B, Basit A et al. A case of methotrexate embryopathy with holoprosencephaly, expanding the phenotype. Birth

defects research (Part A ) Clinical and molecular Teratology .76:138-142 (2006).

8. Buckley LM, Bullaboy CA, Leichtman L, Marquez M . Multiple congenital anomalies associated with weekly low-dose methotrexate treatment of the mother. QJM 1999; 92 (10): 551-563.

9. Berkowitz RS .Subsequent Pregnancy Experience in Patients with Gestational Trophoblastic Disease. The Journal of Reproductive Medicine, 1995. 39, 228-232.

10. Kashimura Y, Kashimura M, Sugimori H, Tsukamoto N, Matsuyama T, Matsukuma K, Kamura T, Saito T, Prophylactic chemotherapy for hydatidiform mole. Five to 15 years follow-up.Cancer. 1986 Aug 1; 58(3):624-9.

11.SP Blagden, MA Foskett, RA Fisher, D Short, S Fuller, ES Newlands and MJ Seckl. The effect of early pregnancy following chemotherapy on disease relapse and foetal outcome in women treated for gestational trophoblastic tumours. British Journal of Cancer.2002; 86: 26 - 30 .

12. Hernandez E, Warner K H. Choriocarcinoma and gestational trophoblastic disease. Effect of chemotherapy for gestational trophoblastic disease on subsequent pregnancy outcome. Obstet GynecolClin.emedicine.medscape.com/article/279116-foll ow-up.Accessed on 16/03/2010 .

113. Kazuhiko I, Takashi M, Fumitaka K, Takanobu S, Nomura S, Shigehiko M. Survival rates of patients with choriocarcinoma treated with chemotherapy without hysterectomy: effects of anticancer agents on subsequent births. Gynecologic Oncology, 2004; 93( 2):529-535

14.Charing Cross Hospital (CXH) Trophoblast Disease Clinic Guide.

www.hmole-chorio.org.uk/clinicians_info_pre_cxh.html accessed on 18/03/2011 